\title{
Circular RNA circRHOT1 promotes hepatocellular carcinoma progression by initiation of NR2F6 expression
}

\author{
Liyan Wang, Haiyan Long, Qinghua Zheng, Xiaotong Bo, Xuhua Xiao and Bin Li* (D)
}

\begin{abstract}
Background: Increasing evidence has revealed a close relationship between non-coding RNAs and cancer progression. Circular RNAs (circRNAs), a recently identified new member of non-coding RNAs, are demonstrated to participate in diverse biological processes, such as development, homeostatic maintenance and pathological responses. The functions of circRNAs in cancer have drawn wide attention recently. Until now, the expression patterns and roles of circRNAs in hepatocellular carcinoma (HCC) have remained largely unknown.

Methods: Bioinformatics method was used to screen differentially expressed novel circRNAs in HCC. Northern blotting, qRT-PCR, in situ hybridization (ISH) and RNA-FISH were utilized to analyzed the expression of circRHOT1 in HCC tisues.CCK8, colony formation, EdU assays were used to analyze proliferation of HCC cells. Transwell assay was utilized to analyze HCC cell migration and invasion. FACS was used for apoptosis analysis. Xenograft experiments were used to analyze tumor growth in vivo. Mass spectrum, RNA pulldown, RIP and EMSA was utilized to test the interaction between circRHOT1 and TIP60. RNA-sequencing method was used to analyze the downstream target gene of circRHOT1.
\end{abstract}

Results: We identified circRHOT1 (hsa_circRNA_102034) as a conserved and dramatically upregulated circRNA in HCC tissues. HCC patients displaying high circRHOT1 level possessed poor prognosis. Through in vitro and in vivo experiments, we demonstrated circRHOT1 significantly promoted HCC growth and metastasis. Regarding the mechanism, we conducted a RNA pulldown with a biotin-labeled circRHOT1-specific probe and found that circRHOT1 recruited TIP60 to the NR2F6 promoter and initiated NR2F6 transcription. Moreover, NR2F6 knockout inhibited growth, migration and invasion, whereas rescuing NR2F6 in circRHOT1-knockout HCC cells rescued the proliferation and metastasis abilities of HCC cells.

Conclusion: Taken together, circRHOT1 inhibits HCC development and progression via recruiting TIP60 to initiate NR2F6 expression, indicating that circRHOT1 and NR2F6 may be potential biomarkers for HCC prognosis.

Keywords: circRHOT1, Hepatocellular carcinoma, TIP60, NR2F6

\section{Background}

Currently, hepatocellular carcinoma (HCC) has become a most prevalent cancer worldwide [1]. This malignancy contributes to a great proportion of cancer-related death each year, especially in China [2]. Until now, the main therapeutic methods for HCC have included curative resection and chemotherapy. In past decades, efforts and advances have been made concerning HCC treatment. However, the prognosis of HCC patients is still very poor, and the 5-year

\footnotetext{
* Correspondence: binlilblb@163.com

Gastrointestinal Department, Affiliated Hospital of Guilin Medical University, Lequn road No.15, Xiufeng district, Guilin 541001, China
}

survival rate is lower than $25 \%$ due to tumor recurrence and metastasis with a high frequency [3]. Therefore, these serious challenges make it very urgent to find novel biomarkers for HCC diagnosis and identify effective therapeutic targets.

Recently, circular RNAs (circRNAs) have attracted much attention. CircRNAs are a new member of non-coding RNAs that have little protein coding potential and make up more than $90 \%$ of the transcriptome in humans $[4,5]$. Circular RNAs are derived from precursor mRNA back splicing and are covalently closed transcripts [6]. Therefore, circRNAs are more stable and resistant to decay machineries 
than linear RNAs [7]. Some studies have shown that the formation of circRNAs relies on the presence of flanked complementary sequences such as Alu elements [8]. Highthroughput sequencing has reveal that the expression of circRNAs is cell, tissue or development specific, suggesting that circRNAs may have specific biological functions [9]. Increasing evidence has shown that circRNAs are potential mediators in tumor biology via diverse mechanisms [10]. For instance, ciRS-7 works as a sponge for miR-7 in brain [11]. circ-Foxo3 induces tumor cell apoptosis by enhancing Foxo3 activity [12]. In HCC, some studies also revealed the existence of numerous circRNAs, such as circMTO1 and CircARSP91 $[7,10]$. However, the function and mechanism of circRNA in HCC need to be further explored.

In our study, we analyzed two circRNA profiles expressed in human HCC tissues and identified circRHOT1 (hsa_circRNA_102034) as a conserved and significantly upregulated circRNA in HCC tissues. Additionally, the expression of circRHOT1 was closely related to the HCC patient prognosis. We found that circRHOT1 could recruit TIP60 to the NR2F6 promoter, initiate NR2F6 expression and eventually promote HCC progression. Thus, circRHOT1 and NR2F6 might act as novel biomarkers for HCC prognosis and promising therapeutic targets.

\section{Methods}

\section{HCC samples}

We obtained 100 paired HCC tissues and adjacent normal tissues from the Affiliated Hospital of Guilin Medical University. The relationship between circRHOT1 expression and clinical severity is listed in Additional file 1: Table S1. Samples receiving chemotherapy or radiotherapy before collection were excluded. Written consent approving the usage of HCC tissues in our study were obtained from each patient. This study was approved by the ethnic committee of the Affiliated Hospital of Guilin Medical University.

\section{Cell culture}

For primary HCC cell culture, the surgical-isolated HCC tissues were digested with collagenase IV and hyaluronidase for $50 \mathrm{~min}$ at $37^{\circ} \mathrm{C}$. Afterwards, the cell suspensions were washed, filtered using a $70 \mu \mathrm{m}$ cell strainer (BD, USA) and seeded on collagen-coated Petri dishes. Fibroblasts and blood vessels were discarded. The primary cancer cells were cultured in DMEM medium supplemented with $20 \% \mathrm{FBS}$, $2 \mathrm{mM}$ glutamine, $1 \mathrm{mM}$ pyruvate, $10 \mathrm{mM}$ HEPES, 100 units $/ \mathrm{mL}$ penicillin/streptomycin, $0.1 \mathrm{mg} / \mathrm{mL}$ gentamicin, and $2 \mathrm{~g} / \mathrm{L}$ Fungizone as previously reported [13]. The primary cells at passage $4 \sim 10$ were used for experiments.

\section{CRISPR-Cas9 knockout system}

circRHOT1, TIP60 or NR2F6 knockout and NR2F6 promoter deletion in HCC cells were established using the CRISPR/Cas9 system as previously described [14, 15].
Briefly, we designed single guide RNAs (sgRNA) using an online tool (http://crispr.mit.edu/) and cloned them into the lenti-Cas9-eGFP vector (Addgene, 63592). Next, 293 $T$ cells were transduced with the lenti-Cas9-eGFP-sgRNA as well as pVSVg (Addgene, 8454), and psPAX2 (Addgene, 12260) to produce lentivirus. The viruses were concentrated with PEG5000 (Sigma Aldrich) and were used to infect HCC cells. For TIP60 or NR2F6 deletion, $\mathrm{GFP}^{+}$cells were isolated by FACS, and the knockout efficiency was verified by western blotting. For circRHOT1 and NR2F6 promoter deletion, two sgRNAs flanking the target sequence were used at the same time. Next, $\mathrm{GFP}^{+}$cells were isolated by FACS, followed by monoclonalization. Their deletion was verified by DNA sequencing. The sgRNA sequences were listed in Additional file 1: Table S2.

\section{Antibodies}

Anti-GAPDH (5174), anti-Cyclin D1 (2978) anti-TIP60 (12058), anti-P21 (2947), and anti-PCNA (13110) were from Cell Signaling Technology. Anti-Flag (F1804) was purchased from Sigma-Aldrich. Anti-NR2F6 (ab137496) was obtained from Abcam.

\section{Xenograft assays}

For in vivo tumor growth, $1 \times 10^{6} \mathrm{HCC}$ cells were subcutaneously injected into the flanks of 5-week-old nude mice from HFK Biosciences ( $n=6$ for each group). The tumor volumes and weights were measured at the indicated time points. Animal assays were approved by the ethnic committee of the Affiliated Hospital of Guilin Medical University.

\section{Cell proliferation assay}

Cell proliferation was measured using CCK- 8 assays as previously reported [16]. Briefly, $1 \times 10^{3}$ cells were seeded in 96-well plates and were cultured for $24 \mathrm{~h}, 48 \mathrm{~h}, 72 \mathrm{~h}$ and $96 \mathrm{~h}$, followed by incubation with $10 \mu \mathrm{l}$ of CCK-8 assay solution in each well for $2 \mathrm{~h}$. The absorbance values at $450 \mathrm{~nm}$ were then measured using an enzyme immunoassay analyzer (Thermo Fisher Scientific, Inc., Waltham, MA, USA). This assay was repeated three times.

\section{Transwell assays}

Cell migration and invasion assays have been described previously [17]. Briefly, $2 \times 10^{3} \mathrm{HCC}$ cells were planted in the upper chamber (Mitrogel (BD) pre-coated for invasion) with $200 \mu \mathrm{l}$ serum-free medium. The lower chamber was added with complete medium. Forty-eight hours later, cells in the lower chamber were fixed and stained with crystal violet $(0.2 \%)$. Cell numbers were determined using a microscope. This assay was repeated three times. 


\section{Real-time quantitative PCR}

TRIzol reagent was used to extract RNA from HCC tissues or cell lines according to the manufacturer's protocol. Then $1 \mu \mathrm{g}$ RNA was used to synthesize cDNA, followed by analysis of gene expression on ABI 7300 qPCR system. Relative expression was normalized to $A C T B$ or U6. The analysis of circRHOT1 was carried out using convergent primers (Forward, 5'-ATCACCATTCCAGCTGATGT-3' and reverse, 5'-TGCTGTCTTTGTCTGTTCTTTC-3') and divergent primers (Forward, 5'-AGTCGATGGATTCCTCTCAT-3' and Reverse, 5'-AAGTTGTTCATCACTCTGTT-3'). The sequences of other primer are listed in Additional file 1: Table S3.

\section{Chromatin immunoprecipitation (ChIP) assay}

ChIP assays were conducted as previously described [15]. Briefly, HCC cells were fixed with $1 \%$ formaldehyde at $37^{\circ} \mathrm{C}$ for $10 \mathrm{~min}$ and lysed. The genome was sonicated to $\sim 500 \mathrm{bp}$ fragments. Anti-TIP60 were added into the lysates and incubated overnight at $4{ }^{\circ} \mathrm{C}$, followed by incubation with Protein A Agarose/Salmon Sperm DNA (50\% Slurry) beads for $4 \mathrm{~h}$. Finally, precipitants were eluted and analyzed by qPCR. Primer sequences were listed in Additional file 1: Table S4.

\section{Electrophoretic mobility shift assay (EMSA)}

For RNA EMSAs, the biotin-labeled circRHOT1 RNA probe was obtained by in vitro transcription using the Biotin RNA Labeling Mix (Roche). The LightShift Chemiluminescent RNA EMSA Kit (Thermo Scientific) was used for the shift assay according to the manufacturers' instructions. This assay was repeated three times.

\section{RNA in situ hybridization}

CircRHOT1 expression in HCC tissues was measured using biotin-labeled circRHOT1 probes. Paraffinized sections were deparaffinized with xylene and $100 \%$ ethanol. The sections were then incubated with biotin-labeled probes for $18 \mathrm{~h}$ at $40^{\circ} \mathrm{C}$. The DAB substrate was used for the colorimetric detection of circRHOT1. Finally, the sections were co-stained with hematoxylin, followed by dehydration in graded alcohols and xylene. Biotin-conjugated probes were purchased from Invitrogen. The CircRHOT1 probe sequence was as follow: 5'-GGAG GAACCTGCTGTCTTTGTCTGTTC-3'. This assay was repeated three times.

\section{Fluorescence in situ hybridization}

Hybridization was performed overnight with circRHOT1 probes. Specimens were analyzed using a Nikon inverted fluorescence microscope. The circRHOT1 probe for fluorescence in situ hybridization (FISH) is $5^{\prime}$-GGAG GAACCTGCTGTCTTTG-3'. This assay was repeated three times.

\section{Northern blotting}

RNAs were isolated from HCC samples using TRIZOL (Invitrogen). CircRHOT1, linear RHOT1 and 18S probes for northern blotting were achieved using the Biotin RNA labeling mix (Roche). The RNA samples were separated by electrophoresis and were transferred to $\mathrm{NC}$ membranes, which were then incubated with the hydration buffer containing the probes. Finally, the RNA signal was detected using the Chemiluminescent Nucleic Acid Detection Module (Thermo Scientific). This assay was repeated three times.

\section{Pulldown and mass spectrometry}

RNA pulldown and mass spectrometry were performed as described before [15]. This assay was repeated three times.

\section{Minigene assay}

This assay was performed according to a previous study [18]. In brief, the sequence of circRHOT1 flanked by two complementary regions (left and right) or only by the left complementary region was inserted into overexpressing vector. Then plasmids were transfected into circRHOT1-depleted $293 \mathrm{~T}$ cells and the level of circRHOT1 was measured by northern blotting.

\section{Analysis of online datasets}

The normalized circRNA expression values in two online-available datasets (GSE94508 and GSE97332) were downloaded from GEO database. Then we first searched differentially expressed circRNAs based on the dataset GSE94508. We selected upregulated circRNAs in tumor tissues according to the dataset GSE94508. Then, we analyzed whether these selected circRNAs were still upregulated in the dataset of GSE97332. Finally, circRNAs that were upregulated in these two datasets were determined. Thus, the most differentially expressed circRNAs between HCC tissues and normal tissues were screened out. Afterwards, we analyzed whether these circRNAs were conserved between human and mouse according to Yang's cohort, which is directly available in their published article [19]. So circRHOT1 was eventually selected. The heatmaps were determined according to the normalized expression levels of circRNAs.

\section{RNA sequencing}

WT and circRHOT1-deficient HCC cells were lysed with TRIZOL reagent, and total RNA was extracted according to the standard method. Next, RNA sequencing was performed (Shenzhen, BGI Genomics Co., Ltd.), and target genes were analyzed. 


\section{Statistical analysis}

All results from at least three independent experiments were analyzed using SPSS software version 20.0 (SPSS Inc., Chicago, IL, USA) or GraphPad Prism 6, and expressed as means $\pm \mathrm{SD}$. One-way analyses of variance, chi-squared tests, and two-tailed Student's t-tests were utilized to calculate statistical significance, as appropriate. Survival rate was determined using Kaplan-Meier method and analyzed with the log-rank test. $P<0.05$ was considered statistically significant.

\section{Results}

\section{CircRHOT1 is overexpressed in HCC tissues}

To identify essential circRNAs expressed in HCC tissues, we analyzed online-available datasets concerning the circRNA profiles in HCC tissues (GSE94508 and GSE97332) [7, 20]. We found that many circRNAs are overexpressed in HCC tissues compared to peritumor tissues (Fig. 1a). Among them, circRHOT1 is one of the most upregulated and conserved circRNAs according to Yang's cohort [19]. Firstly, we validated its features as a circRNA by PCR (Additional file 1: Figure S1a) and RNA sequencing (Additional file 1: Figure S1b). Then, the levels of circRHOT1 in 100 paired HCC tissues and peritumor tissues were determined. Results confirmed that circRHOT1 was significantly upregulated in tumor tissues (Fig. 1b). Next, we chose 5 pairs of HCC sample tissues to subject to northern blotting and validated circRHOT1 was markedly overexpressed in tumor tissue (Fig. 1c). Notably, the linear RHOT1 mRNA level was only slightly elevated as determined by online available datasets and qRT-PCR analysis (Additional file 1: Figure S1c). However, the change on RHOT1 protein level was not obvious in HCC tissues (Additional file 1: Figure S1d). In situ hybridization (ISH) and fluorescence in situ hybridization (FISH) using a biotin-labeled specific probe were further carried out to measure circRHOT1 expression. Results indicated that more circRHOT1 was present in HCC tissues (Fig. 1d, e). In addition, circRHOT1 level was increased in HCC cell lines (Additional file 1: Figure S1e). To investigate the relationship between circRHOT1 level and clinical severity, we checked circRHOT1 expression in HCC tissues with different stages by qRT-PCR. As shown in Fig. 1f, circRHOT1 was expressed more highly in stage III tissues than in stage I/II samples. Moreover, the expression of circRHOT1 in advanced HCC (aHCC) tissues was higher than that in early HCC (eHCC) tissues (Fig. 1g). Next, we investigated the correlation of circRHOT1 expression with the prognosis of HCC patients. We found that HCC patients with higher circRHOT1 expression showed a poorer prognosis by Kaplan-Meier survival analysis (Fig. 1h, i). Taken together, our data suggest that
circRHOT1 is highly expressed in HCC tissues and correlated with a poor prognosis.

\section{CircRHOT1 knockout suppresses HCC cell proliferation, migration and invasion but promotes apoptosis}

We next explored the role of circRHOT1 in HCC progression. A previous study showed that the flanked complementary elements are essential for the formation of circular RNAs [21]. We identified three complementary elements flanking circRHOT1 (Additional file 1: Figure S2a), and only the deletion of the complementary region \#3 in the right flank of circRHOT1 exon 6 abrogated the expression of circRHOT1 as determined by minigene assay (Additional file 1: Figure S2a). Thus, we constructed circRHOT1-deficent HCC cell lines through deleting the region \#3 in the right flank of circRHOT1 by CRISPR/Cas9 technology. We confirmed the efficiency of circRHOT1 deletion by PCR (Additional file 1: Figure S2b), Northern blotting (Additional file 1: Figure S2c) and qRT-PCR (Fig. 2a). Notably, circRHOT1 deletion did not affect the expression of RHOT1 mRNA and protein (Additional file 1: Figure S2c, d). Then, we performed CCK-8, colony formation and EdU incorporation assays to assess the effect of circRHOT1 on cell proliferation. As shown, circRHOT1 knockout significantly inhibited cell proliferation, colony formation and EdU incorporation (Fig. 2b-d). Next, we conducted Transwell assays and found that circRHOT1 knockout significantly suppressed the migration and invasion of HCC cells (Fig. 2e, f). Moreover, circRHOT1 deletion dramatically promoted the apoptotic cell percentage (Fig. 2g), which is further confirmed by the detection of Caspase 3/7 Activity (Fig. 2h). To further validate the effects of circRHOT1 on HCC cells, we silenced its expression in HCC cell lines, including Hep3B and Huh7 cells (Additional file 1: Figure S2e). Functional experiments from CCK8, colony formation, EdU incorporation, Transwell and FACS results also demonstrated that circRHOT1 knockdown suppressed proliferation, migration and invasion but promoted apoptosis in HCC cells (Additional file 1: Figure S2f-k). Additionally, we conducted rescue assay by re-expressing circRHOT1 in circRHOT1-deleted HCC cells (Additional file 1: Figure S2l). A series of experiments indicated that restoration of circRHOT1 rescued the effects of circRHOT1 knockout on HCC cell proliferation, migration, invasion and survival (Additional file 1: Figure S2m-q), indicating the essential role of circRHOT1 in HCC.

To evaluate the function of circRHOT1 in vivo, we performed xenograft experiments with WT and circRHOT1-deleted HCC cells. As shown in Fig. 2i, circRHOT1 knockout significantly reduced the tumor weight. Furthermore, xenograft experiments with circRHOT1 low- or high-expressed HCC cells showed that circRHOT1 overexpression promoted tumor growth in vivo (Fig. 2j). Collectively, the above data convincingly 


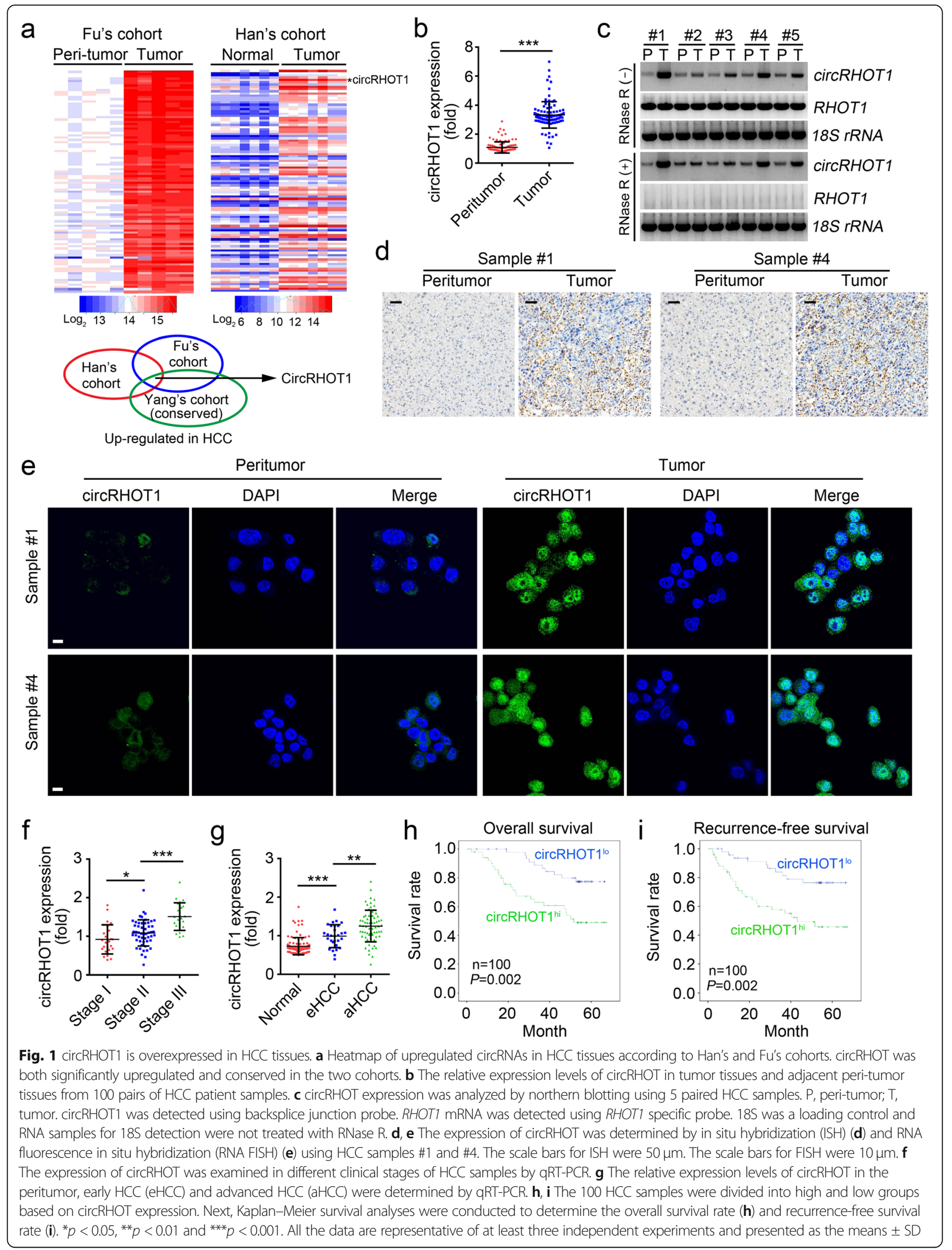




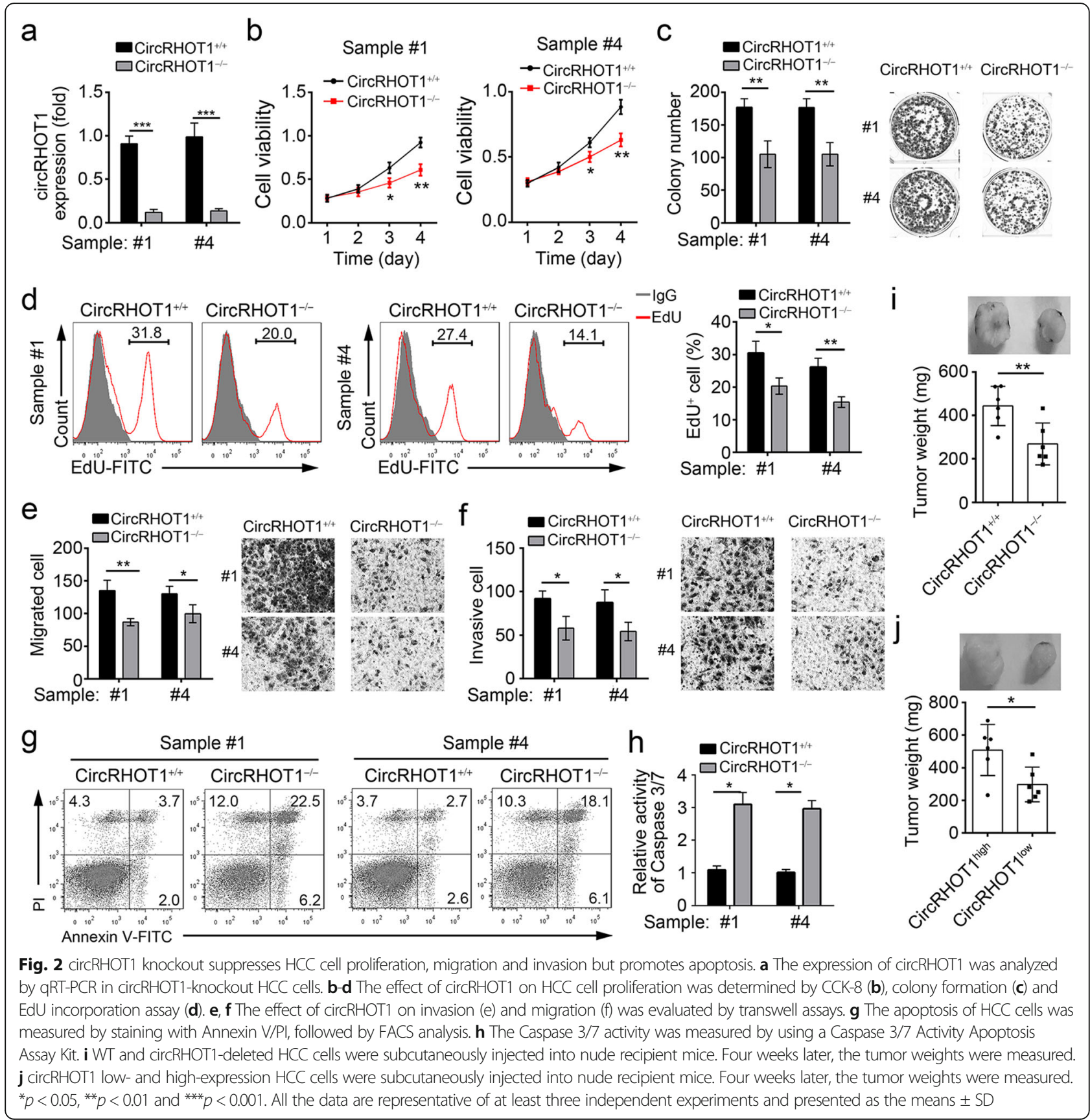

demonstrated that circRHOT1 promotes HCC development and progression.

\section{CircRHOT1 regulates NR2F6 expression in HCC cells}

To further investigate the underlying molecular mechanism, we analyzed the differentially expressed genes after circRHOT1 deletion using RNA sequencing. Many genes were downregulated, among which NR2F6 is the most downregulated and possesses high expression value in HCC cells (Fig. 3a). We chose seven downregulated genes based on fold-change and expression level in WT HCC cells to verify the RNA sequencing results by qRT-PCR (Fig. 3b). Notably, these seven genes were also downregulated in Hep3B and Huh7 cells after circRHOT1 silence (Additional file 1: Figure S3a). Western blotting indicated NR2F6 protein level was significantly decreased in circRHOT1-deleted HCC cells (Fig. 3c) and circRHOT1-silenced HCC cell lines (Additional file 1: Figure S3b). Our above data showed that circRHOT1 is mainly distributed in the nucleus (Fig. 1d, e). To determine whether circRHOT1 regulates NR2F6 transcription, we performed chromatin isolation by RNA purification (CHIRP) with 


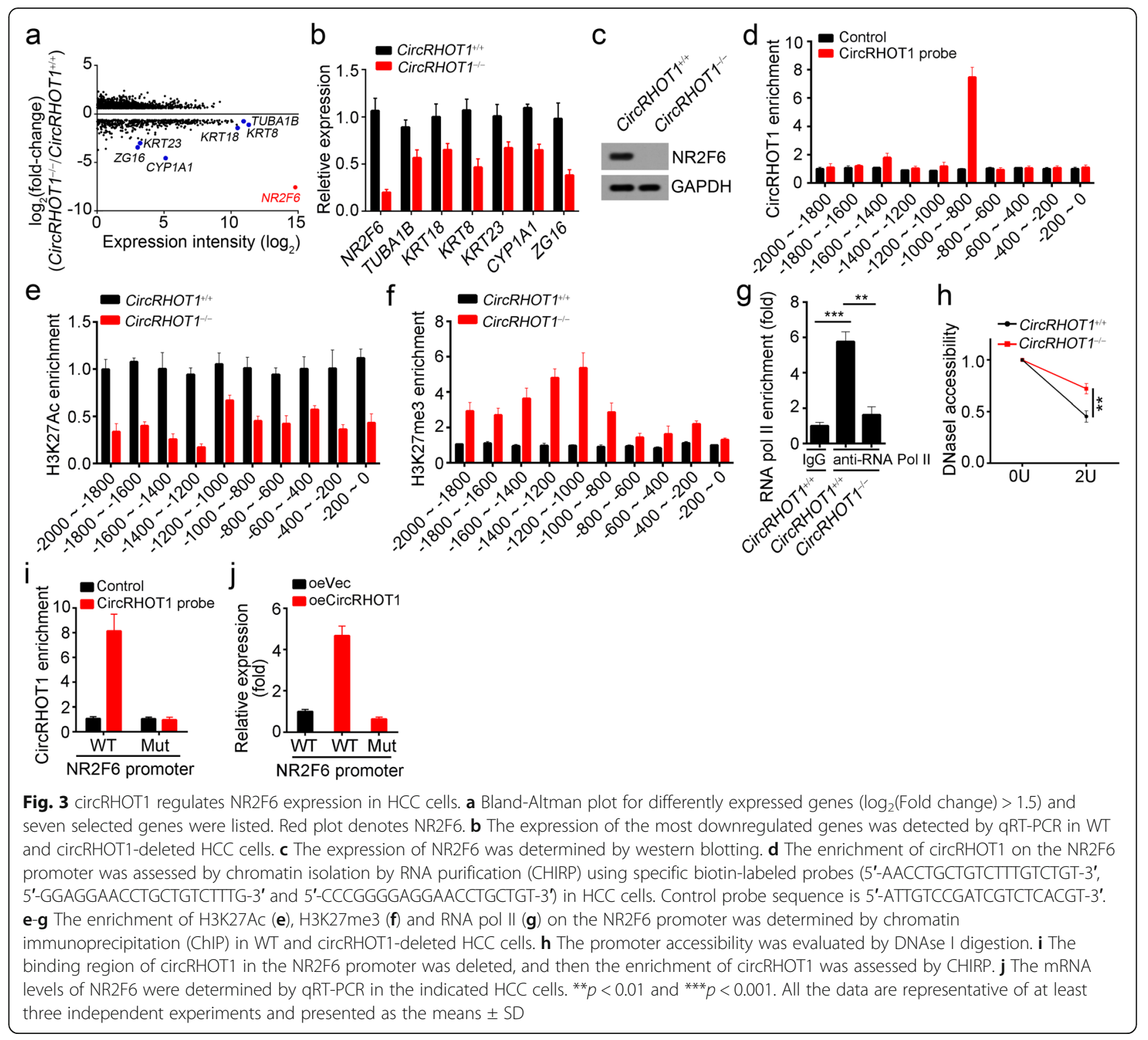

specific biotin-labeled circRHOT1 probe and found that circRHOT1 could be enriched on the region of - 1000 $800 \mathrm{bp}$ from the transcription start site (TSS) of NR2F6 promoter (Fig. 3d). Moreover, the enrichment of the histone active modification H3K27Ac was reduced after circRHOT1 knockout while that of H3K27me3 was increased (Fig. 3e, f). Consistently, RNA pol II could not bind to the NR2F6 promoter after circRHOT1 deletion (Fig. 3g). Moreover, the promoter of NR2F6 in circRHOT1-deleted HCC cells was more resistant to DNaseI digestion (Fig. 3h), suggesting that circRHOT1 is indispensable for accessibility of the NR2F6 promoter. To further demonstrate the role of the circRHOT1 binding region on NR2F6 promoter, we deleted this region in HCC cells. CircRHOT1 could not enrich on the NR2F6 promoter after this region deletion (Fig. 3i). Additionally, we found that the overexpression of
circRHOT1 promoted the mRNA level of NR2F6, while deletion of the binding region abrogated this effect (Fig. 3j). Thus, circRHOT1 was associated with the NR2F6 promoter and promoted its expression.

\section{CircRHOT1 is associated with TIP60}

To investigate whether circRHOT1 cooperated with specific proteins to regulate NR2F6 expression, we performed RNA pulldown using HCC cell lysates, followed by silver staining and mass spectrum identification. We identified TIP60 as a potential interactive protein of circRHOT1 (Fig. 4a). Furthermore, RNA pulldown with TIP60-overexpressing HCC cells showed that biotin-labeled circRHOT1 precipitated Flag-TIP60 (Fig. 4b). Moreover, biotin-labeled circRHOT1 probe could precipitate endogenous TIP60 in WT HCC cell lysates (Fig. 4c). Besides, TIP60-specific 


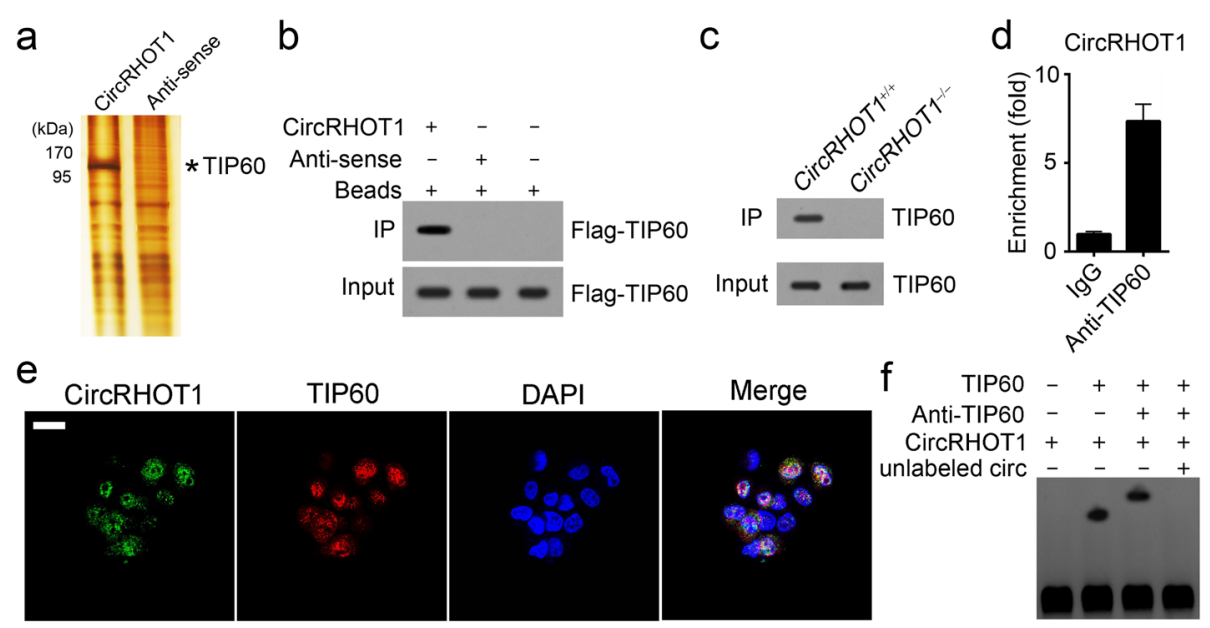

Fig. 4 CircRHOT1 associates with TIP60. a A specific biotin-labeled circRHOT1 probe was added to HCC lysates, and the precipitated proteins were resolved by SDS-PAGE, followed by silver staining. Next, the differential band in the circRHOT1 probe lane was identified by mass spectrometry (MS). The anti-sense of circRHOT1 serves as control. As shown, TIP60 was identified. b RNA pulldown was performed to verify the interaction between TIP60 and circRHOT1 using TIP60-overexpressing HCC cells. Biotin-labeled circRHOT1 or anti-sense was used to precipitate Flag-TIP60. c The specific biotinlabeled circRHOT1 probe (5'-TCTTCTGCCCGGGGAGGAACCTGCTGTCTTTGTCTGTTCTTCAT-3') did not precipitate TIP60 in circRHOT1-knockout HCC cells. d RNA IP results indicated that TIP60 enriched circRHOT1 in HCC cells. e CircRHOT1 co-localized with TIP60 in HCC cells as shown by RNA fluorescence in situ hybridization (FISH). Scale bar, $10 \mu \mathrm{m}$. f RNA EMSA assay indicated that TIP60 interacts with circRHOT1 directly. Linear circRHOT1 was labeled with biotin. All the data are representative of at least three independent experiments and presented as the means \pm SD

antibodies enriched endogenous circRHOT1 in HCC cells (Fig. 4d). According to FISH assay, circRHOT1 was co-localized with TIP60 in HCC cells (Fig. 4e). RNA-EMSA assay also demonstrated that TIP60 directly interacts with circRHOT1 (Fig. 4f).

\section{CircRHOT1 recruits TIP60 to initiate NR2F6 expression}

To further determine whether TIP60 participates in the regulation of NR2F6 expression, we performed ChIP assays with HCC cells. We found that TIP60 was enriched in the same region of the NR2F6 promoter as circRHOT1 (Fig. 5a). Furthermore, TIP60 knockout also inhibited the enrichment of H3K27Ac and RNA pol II on the NR2F6 promoter (Fig. 5b, c). In addition, TIP60 deletion downregulated the mRNA and protein levels of NR2F6 in HCC cells (Fig. 5d, e). Next, we wanted to determine the role of circRHOT1 in TIP60-mediated expression of NR2F6. We found that circRHOT1 knockout abrogated the enrichment of TIP60 on the promoter of NR2F6 (Fig. 5f). DNA FISH also indicated that circRHOT1 knockout abolished the co-localization of TIP60 with the NR2F6 promoter (Fig. $5 \mathrm{~g}$ ). Importantly, circRHOT1 deficiency lost the recruitment of the NuA4 complex to the NR2F6 promoter, whereas the NuA4 complex bound to the NR2F6 promoter in control HCC cells (Fig. 5h). Moreover, double knockout of circRHOT1 and TIP60 further inhibited the mRNA level of NR2F6 in HCC cells (Fig. 5i). We then analyzed the relationships among the expression levels of circRHOT1, TIP60 and NR2F6 in HCC tissues. We found that the protein and mRNA levels of NR2F6 were positively correlated with those of TIP60 in HCC tissues by qRT-PCR and according to the GSE45436 dataset (Fig. 5j, k). Additionally, there was a positive correlation between the expression of NR2F6 and circRHOT1 in HCC tissues (Fig. 5k and Additional file 1: Figure S3c) while no significant expression correlation between circRHOT1 and TIP60 existed (Additional file 1: Figure S3c). Altogether, circRHOT1 is essential for TIP60-mediated NR2F6 expression in HCC tissues.

\section{Restoration of NR2F6 rescues circRHOT1 deletion-} mediated suppression of HCC growth and metastasis To further explore the role of NR2F6, we examined its expression in HCC tissues. According to Park's cohort (GSE36376) [22] and Zhang's cohort (GSE25097) [23-25], NR2F6 was upregulated in HCC tissues compared with that in adjacent normal tissues (Fig. 6a, b). Furthermore, we examined the expression of NR2F6 in 100 paired HCC samples and found that NR2F6 was upregulated in tumor tissues (Fig. 6c). Immunohistochemical analysis and western blotting confirmed that NR2F6 level was elevated in HCC tissues (Fig. 6d, e). Moreover, higher expression of NR2F6 in HCC patients suggested a poorer prognosis (Fig. 6f). We then knocked out NR2F6 or restored its protein level in circRHOT1-deleted HCC cells (Fig. 6g). CCK-8 and Transwell assays revealed that knockout of either circRHOT1 or NR2F6 inhibited cell proliferation, migration and invasion, while restoration of NR2F6 in circRHOT1deleted HCC cells rescued cell proliferation, migration and 


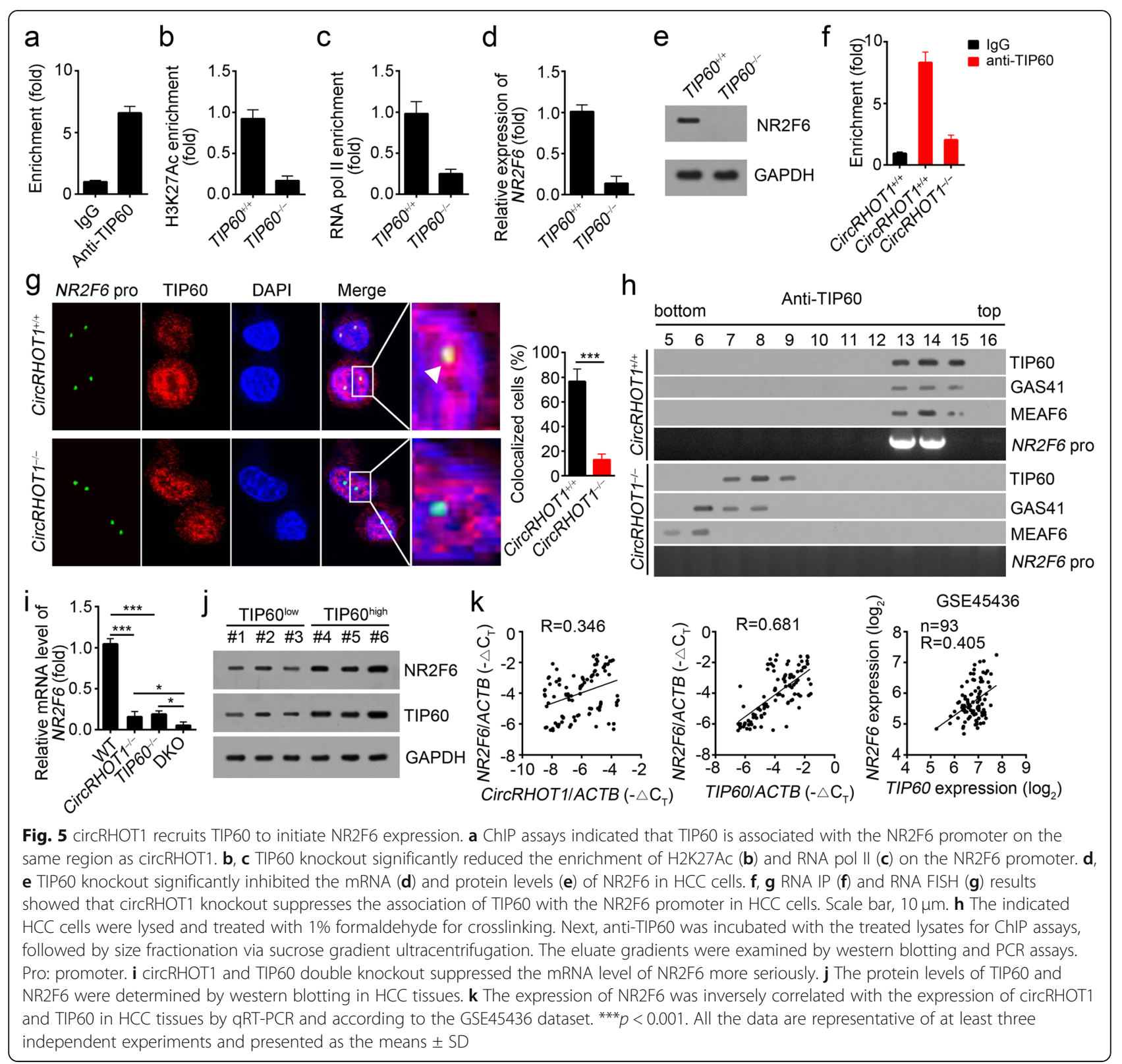

invasion in vitro (Fig. 6h-j). In vivo xenograft experiments showed that knockout of circRHOT1 or NR2F6 suppressed tumor growth. However, restoration of NR2F6 rescued circRHOT1-deletion-mediated inhibition on tumor growth (Fig. 6k). Thus, our results demonstrated that circRHOT1 inhibits HCC progression via the activation of NR2F6.

\section{Discussion}

With the advancement on high-throughput sequencing technology, increasing circRNAs have been identified in human tissues [26]. The study concerning the functions of circRNAs has attracted increasing attention. Many reports have revealed a close correlation between circRNA expression and human diseases, especially in tumorigenesis
$[27,28]$. However, the functions of circRNAs in HCC remain elusive. In this study, we screened out an uncharacteristic and upregulated circRNA named circRHOT1 in HCC using a bioinformatics method. Additionally, we investigated the function and mechanism of circRHOT1 during $\mathrm{HCC}$ development.

Currently, many molecules, including proteins and noncoding RNAs, have been identified as potential biomarkers for HCC diagnosis or prognosis [29]. However, whether circular RNAs could be good biomarkers remains to be further investigated. In our study, we showed that circRHOT1 expression was upregulated in HCC tissues. And increased expression of circRHOT1 in HCC patients predicted a poor prognosis. Therefore, circRHOT1 may serve as a potential 


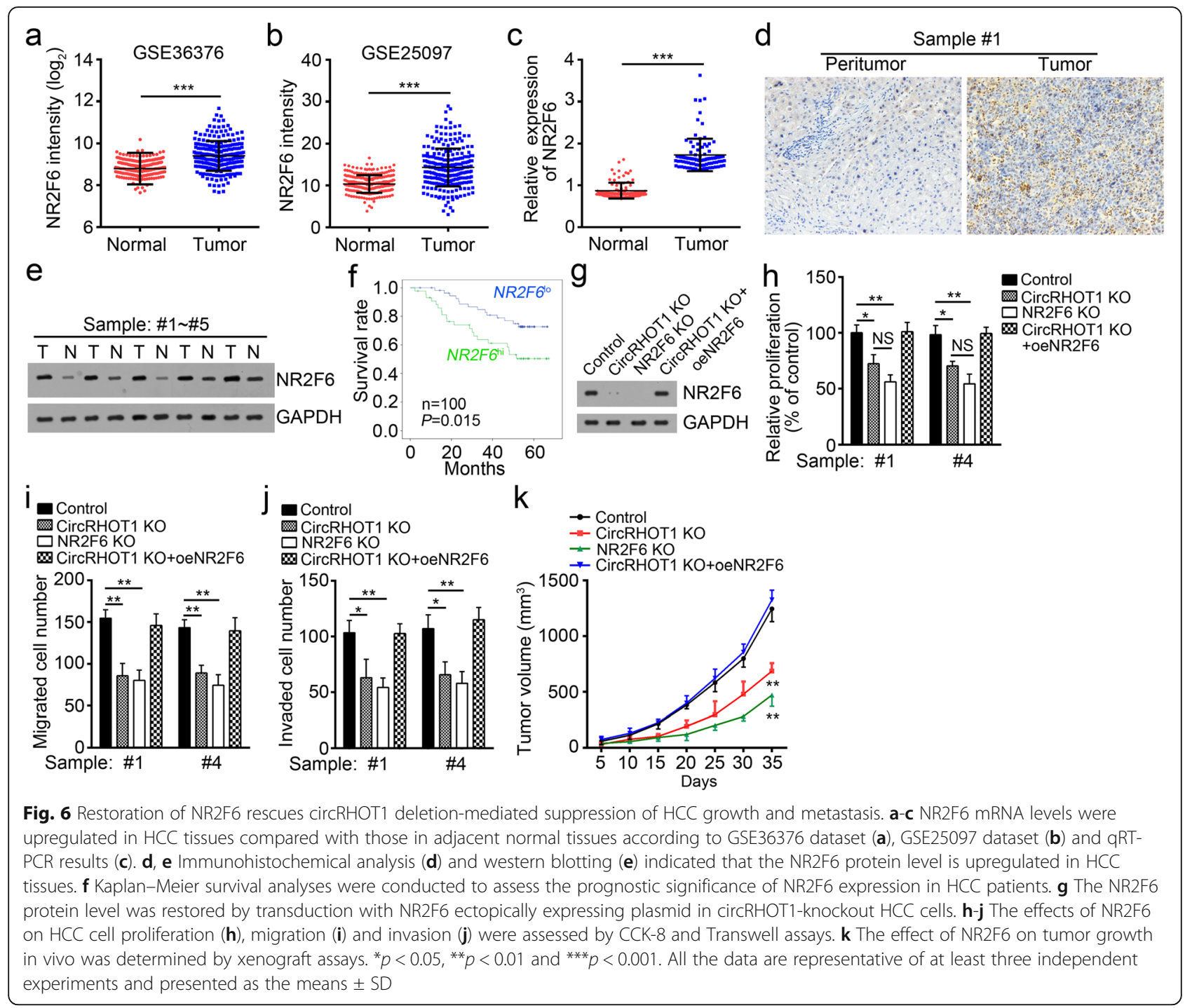

biomarker for HCC prognosis. Our results were from 100 HCC patients. Thus, to determine the practical value of circRHOT1 expression for evaluating HCC prognosis still needs a larger sample size and further investigation. Additionally, whether circRHOT1 might be a marker for HCC diagnosis must be explored. A previous study showed that circular RNA is stable and exists in exosomes [30]. Whether circRHOT1 is present in exosomes and whether circulating circRHOT1 is also related to HCC remain to be explored. CircRHOT1 is originated from RHOT1 mRNA. However, we found that RHOT1 mRNA level was slightly upregulated in HCC tissues compared to normal tissues, according to online datasets (GSE36376 and GSE25097) and qRT-PCR analysis. Notably, circRHOT1 level was upregulated in HCC tissues over four times (Fig. 1b). Thus, upregulation of circRHOT1 in HCC tissues might be due to both the aberrant transcription of RHOT mRNA itself and the frequent backsplicing event, which requires further validation.
The molecular mechanisms that regulate cancer development and progression have not been fully elaborated. Additionally, how circRNAs participate in tumorigenesis requires further investigation. Many studies have shown that circRNAs could regulate the expression of oncogenes or tumor suppressors mainly via a circRNA-miRNA-mRNA axis [31, 32]. A few reports have also shown that circRNAs may associate with specific proteins to exert important functions in cancer $[33,34]$. The regulatory network of circRNAs with other molecules requires more investigation in the process of tumor development. In our study, we showed that circRHOT1 mainly locates in the nucleus. Therefore, we hypothesized whether circRHOT1 interacts with some proteins to promote $\mathrm{HCC}$ progression. By MS identification and RNA pulldown, we verified that circRHOT1 associates with TIP60. TIP60 is the major subunit of the NuA4 chromatin remodeling complex, which regulates histone acylation modification and initiates the expression of target 
genes [35]. Previous studies indicate that TIP60 promotes mammary tumorigenesis [36], pleural mesothelioma malignance [37] and prostate cancer growth [38]. TIP60 is also involved in DNA repair [39]. Whether TIP60 participates in liver cancer remains unclear. Hence, we further explored the mechanism by which circRHOT1 and TIP60 regulate the expression of specific downstream target genes.

By RNA sequencing, we analyzed the downstream regulated genes by circRHOT1. We identified NR2F6 as the most downregulated gene after circRHOT1 knockout. NR2F6 is a member of the NR2F family, which could dimerize with RXR/NR2B1 or other NRs and recognize various DNA response elements [40]. A previous study has indicated that NR2F6 is a regulator of adaptive immunity ${ }^{31}$. NR2F6 regulates $\mathrm{CD}_{4}^{+}$T-cell effector functions and negatively controls cell development [41, 42]. Recent reports showed that NR2F6 is involved in human cancers such as ovarian cancer and cervical cancer [43, 44]. However, the functional role of NR2F6 has not been investigated in HCC. In this study, we demonstrated that NR2F6 is the target gene of circRHOT1 and TIP60 in HCC. By ChIP and FISH assays, we proved that circRHOT1 recruited TIP60 to the NR2F6 promoter, which leads to further recruitment of other components of NuA4 complex by TIP60 and eventually initiated NR2F6 expression. Moreover, we showed that NR2F6 was overexpressed in HCC tissues. Knocking out NR2F6 significantly inhibited HCC growth and metastasis. We also found that higher expression of NR2F6 in HCC patients predicts lower survival rate, indicating NR2F6 might be a prognostic marker. Additionally, restoration of NR2F6 in circRHOT1-deficient HCC cells rescued cell proliferation, migration and invasion. Thus, our study expanded the role of circRNAs and its functional mechanism in HCC. Notably, we observed that either circRHOT1 knockout or NR2F6 knockout suppressed the activation of NOTCH2 pathway (Additional file 1: Figure S3d), suggesting circRHOT1/ TIP60/NR2F6 axis might exert roles through NOTCH2 signaling partially. Even so, the downstream mechanism of NR2F6 in HCC requires more exploration. Due to the importance of NR2F6 in other types of cancers [43, 44], whether circRHOT1/TIP60/NR2F6 axis also plays a role in these cancers needs further investigation.

\section{Conclusion}

In summary, our findings suggested that circRHOT1 is highly expressed in HCC tissues and correlated with HCC patients' prognosis. Mechanistically, circRHOT1 promotes HCC development and progression via TIP60-dependent NR2F6 expression (Additional file 1: Figure S3e). Our results suggest that circRHOT1 may serve as a potential biomarker for HCC prognosis. Notably, due to the difficulty of inhibiting circRHOT1 in HCC patients, it might be more promising to pharmacologically inhibit NR2F6 as a therapeutic target.

\section{Additional file}

Additional file 1: Additional informations. (DOCX 1045 kb)

\begin{abstract}
Abbreviations
ceRNA: Competing endogenous RNA; circRNA: Circular RNA; FISH: Fluorescence in situ hybridization; GC: Gastric cancer; IHC: Immunohistochemistry; ISH: In situ hybridization; qRT-PCR: Real-time quantitative polymerase chain reaction; RIP: RNA immunoprecipitation
\end{abstract}

\section{Acknowledgements}

We thank all patients involved in this study.

\section{Authors' contributions}

$\mathrm{BL}$ and $\mathrm{LW}$ conceived the study and performed the whole experimental work in its design and coordination. $\mathrm{HL}, \mathrm{QZ}$ and $\mathrm{XB}$ participated in parts of the experiments and analysis of the data. XX contributed to provide clinical samples. The manuscript was written by LW, and revised by BL. All authors read and approved the final manuscript.

\section{Funding}

This work was supported by Grant from Department of Health of Guangxi Zhuang Autonomous Region (S201316-06).

\section{Availability of data and materials}

The microarray data used in the study (GSE94508, GSE97332, GSE36376 and GSE25097) are available in a public repository from GEO database. The RNAsequencing raw data and normalized results were submitted to the SRA database (SRP165820) and GEO database (GSE121468).

Ethics approval and consent to participate

The present study was approved by the Ethics Committee of Affiliated Hospital of Guilin Medical University.

Consent for publication

Consent was achieved from all patients.

\section{Competing interests}

The authors declare that they have no competing interests.

Received: 25 January 2019 Accepted: 10 July 2019

Published online: 19 July 2019

References

1. Torre LA, Bray F, Siegel RL, Ferlay J, Lortet-Tieulent J, Jemal A. Global cancer statistics, 2012. CA Cancer J Clin. 2015;65:87-108.

2. Jemal A, Bray F, Center MM, Ferlay J, Ward E, Forman D. Global cancer statistics. CA Cancer J Clin. 2011;61:69-90.

3. El-Serag HB. Hepatocellular carcinoma. N Engl J Med. 2011;365:1118-27.

4. Consortium EP. An integrated encyclopedia of DNA elements in the human genome. Nature. 2012;489:57-74.

5. Sana J, Faltejskova P, Svoboda M, Slaby O. Novel classes of non-coding RNAs and cancer. J Transl Med. 2012;10:103.

6. Chen LL. The biogenesis and emerging roles of circular RNAs. Nat Rev Mol Cell Biol. 2016;17:205-11.

7. Han D, Li JX, Wang HM, Su XP, Hou J, Gu Y, Qian C, Lin Y, Liu X, Huang MY, et al. Circular RNA circMTO1 acts as the sponge of MicroRNA-9 to suppress hepatocellular carcinoma progression. Hepatology. 2017;66:1151-64.

8. Jeck WR, Sorrentino JA, Wang K, Slevin MK, Burd CE, Liu JZ, Marzluff WF, Sharpless NE. Circular RNAs are abundant, conserved, and associated with ALU repeats. RNA. 2013;19:141-57.

9. Yao T, Chen QQ, Fu LY, Guo JM. Circular RNAs: biogenesis, properties, roles, and their relationships with liver diseases. Hepatol Res. 2017;47:497-504.

10. Shi L, Yan PJ, Liang YL, Sun Y, Shen JL, Zhou SJ, Lin H, Liang X, Cai XJ. Circular RNA expression is suppressed by androgen receptor (AR)-regulated adenosine deaminase that acts on RNA (ADAR1) in human hepatocellular carcinoma. Cell Death Dis. 2017;8:e3171.

11. Hansen TB, Jensen TI, Clausen BH, Bramsen JB, Finsen B, Damgaard CK, Kjems J. Natural RNA circles function as efficient microRNA sponges. Nature. 2013:495:384-8. 
12. Du WW, Fang L, Yang WN, Wu N, Awan FM, Yang ZG, Yang BB. Induction of tumor apoptosis through a circular RNA enhancing Foxo3 activity. Cell Death Differ. 2017;24:357-70.

13. Liu M, Gu P, Guo WJ, Fan XW. C6 ceramide sensitizes the anti-hepatocellular carcinoma (HCC) activity by AZD-8055, a novel mTORC1/2 dual inhibitor. Tumor Biol. 2016;37:11039-48.

14. Joung J, Konermann S, Gootenberg JS, Abudayyeh OO, Platt RJ, Brigham MD, Sanjana NE, Zhang F. Genome-scale CRISPR-Cas9 knockout and transcriptional activation screening. Nat Protoc. 2017;12:828-63.

15. Zhu P, Wang Y, Wu J, Huang G, Liu B, Ye B, Du Y, Gao G, Tian Y, He L, Fan Z. LncBRM initiates YAP1 signalling activation to drive self-renewal of liver cancer stem cells. Nat Commun. 2016;7:13608.

16. Dou CQ, Sun LY, Jin X, Han MM, Zhang B, Jiang X, Lv JY, Li T. Long non-coding RNA CARLo-5 promotes tumor progression in hepatocellular carcinoma via suppressing miR-200b expression. Oncotarget. 2017;8:70172-82.

17. Zhu L, Yang N, Chen J, Zeng T, Yan S, Liu Y, Yu G, Chen Q, Du G, Pan W, et al. LINC00052 upregulates EPB41L3 to inhibit migration and invasion of hepatocellular carcinoma by binding miR-452-5p. Oncotarget. 2017;8:63724.

18. Wang Y, Wang Z. Efficient backsplicing produces translatable circular mRNAs. RNA. 2015;21:172-9.

19. Dong R, Ma XK, Chen LL, Yang L. Increased complexity of circRNA expression during species evolution. RNA Biol. 2017;14:1064-74.

20. Fu LY, Yao T, Chen QQ, Mo XY, Hu YR, Guo JM. Screening differential circular RNA expression profiles reveals hsa_ circ_ 0004018 is associated with hepatocellular carcinoma. Oncotarget. 2017;8:58405-16.

21. Zhang XO, Wang HB, Zhang Y, Lu XH, Chen LL, Yang L. Complementary sequence-mediated exon circularization. Cell. 2014;159:134-47.

22. Lim HY, Sohn I, Deng S, Lee J, Jung SH, Mao M, Xu J, Wang K, Shi S, Joh JW, et al. Prediction of disease-free survival in hepatocellular carcinoma by gene expression profiling. Ann Surg Oncol. 2013;20:3747-53.

23. Tung EK, Mak CK, Fatima S, Lo RC, Zhao H, Zhang C, Dai H, Poon RT, Yuen MF, Lai CL, et al. Clinicopathological and prognostic significance of serum and tissue Dickkopf-1 levels in human hepatocellular carcinoma. Liver Int. 2011;31:1494-504.

24. Sung WK, Zheng H, Li S, Chen R, Liu X, Li Y, Lee NP, Lee WH, Ariyaratne PN, Tennakoon C, et al. Genome-wide survey of recurrent HBV integration in hepatocellular carcinoma. Nat Genet. 2012:44:765-9.

25. Lamb JR, Zhang C, Xie T, Wang K, Zhang B, Hao K, Chudin E, Fraser HB, Millstein J, Ferguson $M$, et al. Predictive genes in adjacent normal tissue are preferentially altered by sCNV during tumorigenesis in liver cancer and may rate limiting. PLoS One. 2011;6:e20090.

26. Caiment F, Gaj S, Claessen S, Kleinjans J. High-throughput data integration of RNA-miRNA-circRNA reveals novel insights into mechanisms of benzo[a]pyrene-induced carcinogenicity. Nucleic Acids Res. 2015;43:2525-34.

27. Yang X, Yuan W, Tao J, Li P, Yang C, Deng X, Zhang X, Tang J, Han J, Wang $J$, et al. Identification of circular RNA signature in bladder cancer. J Cancer. 2017:8:3456-63.

28. Liu W, Zhang J, Zou C, Xie X, Wang Y, Wang B, Zhao Z, Tu J, Wang X, Li H, et al. Microarray expression profile and functional analysis of circular RNAs in osteosarcoma. Cell Physiol Biochem. 2017;43:969-85.

29. Dai M, Chen S, Wei X, Zhu X, Lan F, Dai S, Qin X. Diagnosis, prognosis and bioinformatics analysis of IncRNAs in hepatocellular carcinoma. Oncotarget. 2017:8:95799-809.

30. Li Y, Zheng QP, Bao CY, Li SY, Guo WJ, Zhao J, Chen D, Gu JR, He XH, Huang SL. Circular RNA is enriched and stable in exosomes: a promising biomarker for cancer diagnosis. Cell Res. 2015;25:981-4.

31. Huang XY, Huang ZL, Xu YH, Zheng Q, Chen Z, Song W, Zhou J, Tang ZY, Huang XY. Comprehensive circular RNA profiling reveals the regulatory role of the circRNA-100338/miR-141-3p pathway in hepatitis B-related hepatocellular carcinoma. Sci Rep. 2017;7:5428.

32. Bai N, Peng E, Qiu X, Lyu N, Zhang Z, Tao Y, Li X, Wang Z. circFBLIM1 act as a ceRNA to promote hepatocellular cancer progression by sponging miR346. J Exp Clin Cancer Res. 2018;37:172.

33. Du WW, Yang WN, Liu E, Yang ZG, Dhaliwal P, Yang BB. Foxo3 circular RNA retards cell cycle progression via forming ternary complexes with p21 and CDK2. Nucleic Acids Res. 2016;44:2846-58.

34. Yang Q, Du WW, Wu N, Yang WN, Awan FM, Fang L, Ma J, Li XM, Zeng Y, Yang ZG, et al. A circular RNA promotes tumorigenesis by inducing c-myc nuclear translocation. Cell Death Differ. 2017;24:1609-20.

35. Xu P, Li CM, Chen ZH, Jiang SY, Fan SL, Wang JW, Dai JB, Zhu P, Chen ZC. The NuA4 core complex acetylates nucleosomal histone $\mathrm{H} 4$ through a double recognition mechanism. Mol Cell. 2016;63:965-75.
36. Bassi C, Li YT, Khu K, Mateo F, Baniasadi PS, Elia A, Mason J, Stambolic V, Pujana MA, Mak TW, Gorrini C. The acetyltransferase Tip60 contributes to mammary tumorigenesis by modulating DNA repair. Cell Death Differ. 2016;23:1198-208.

37. Cregan S, McDonagh L, Gao Y, Barr MP, O'Byrne K, Finn SP, Cuffe S, Gray SG. KAT5 (Tip60) is a potential therapeutic target in malignant pleural mesothelioma. Int J Oncol. 2016;48:1290-6.

38. Shiota M, Yokomizo A, Masubuchi D, Tada Y, Inokuchi J, Eto M, Uchiumi T, Fujimoto N, Naito S. Tip60 promotes prostate cancer cell proliferation by translocation of androgen receptor into the nucleus. Prostate. 2010;70:540-54.

39. Ikura M, Furuya K, Matsuda S, Matsuda R, Shima H, Adachi J, Matsuda T, Shiraki T, Ikura T. Acetylation of histone H2AX at Lys 5 by the TIP60 histone acetyltransferase complex is essential for the dynamic binding of NBS1 to damaged chromatin. Mol Cell Biol. 2015;35:4147-57.

40. Klepsch V, Gerner RR, Klepsch S, Olson WJ, Tilg H, Moschen AR, Baier G, Hermann-Kleiter N. Nuclear orphan receptor NR2F6 as a safeguard against experimental murine colitis. Gut. 2018;67:1434-44.

41. Hermann-Kleiter N, Baier G. Orphan nuclear receptor NR2F6 acts as an essential gatekeeper of Th17 CD4(+) T cell effector functions. Cell Commun Signal. 2014;12:38.

42. Ichim CV, Dervovic DD, Zuniga-Pflucker JC, Wells RA. The orphan nuclear receptor Ear-2 (Nr2f6) is a novel negative regulator of T cell development. Exp Hematol. 2014;42:46-58.

43. Liu J, Li T, Liu XL. DDA1 is induced by NR2F6 in ovarian cancer and predicts poor survival outcome. Eur Rev Med Pharmacol Sci. 2017;21:1206-13.

44. Niu CH, Sun XY, Zhang WJ, Li H, Xu LQ, Li J, Xu BK, Zhang YN. NR2F6 expression correlates with pelvic lymph node metastasis and poor prognosis in early-stage cervical cancer. Int J Mol Sci. 2016;17:1694.

\section{Publisher's Note}

Springer Nature remains neutral with regard to jurisdictional claims in published maps and institutional affiliations.

Ready to submit your research? Choose BMC and benefit from:

- fast, convenient online submission

- thorough peer review by experienced researchers in your field

- rapid publication on acceptance

- support for research data, including large and complex data types

- gold Open Access which fosters wider collaboration and increased citations

- maximum visibility for your research: over $100 \mathrm{M}$ website views per year

At BMC, research is always in progress.

Learn more biomedcentral.com/submissions 